\title{
Graph Based Semi and Unsupervised Classification and Segmentation of Microscopic Images
}

\author{
Vinh Thong Ta, Olivier Lezoray, Abderrahim Elmoataz \\ Université de Caen Basse-Normandie, Laboratoire GREYC CNRS UMR 6072, Équipe Image \\ 6 Boulevard Maréchal Juin, F-14050 Caen Cedex, France \\ vinhthong.ta@unicaen.fr, olivier.lezoray@unicaen.fr, abder.elmoataz@greyc.ensicaen.fr
}

\begin{abstract}
In this paper, we propose a general formulation of discrete functional regularization on weighted graphs. This framework can be used on any multi-dimensional data living on graphs of the arbitrary topologies. In this work, we focus on microscopic image segmentation and classification within semi and unsupervised schemes. Moreover, to provide a fast image segmentation we propose a graph based image simplification as a pre-processing step. Biological elements contained in images such as cells, cytoplasm and nuclei are segmented and classified with this image simplification and label diffusion processes on weighted graphs.

Keywords-Discrete regularization, weighted graphs, microscopic images, image simplification, semi-supervised, unsupervised, segmentation, classification.
\end{abstract}

\section{INTRODUCTION}

Microscopic image segmentation consists in extracting cells from image background and nuclei from cytoplasm, i.e. localizing homogenous regions relative to image content. Then, the classification step corresponds to classify extracted elements in the same biological groups, i.e. to group similar objects into the same class among the existing ones in the image.

Numerous image segmentation and classification methods can be found in the literature. Among existing ones, derivative approaches from supervised to unsupervised machine learning algorithms or variational methods have been successfully applied in computer vision problems. Variational methods, based on regularization, provide a framework to handle image processing problems by designing and solving Partial Differential Equations (PDEs) in continuous domain. Then, PDEs are discretized in order to fit with the image domain. Many PDE-based resolution schemes have been presented so far in the literature (see, for instance [1], [2], and references therein for more details on variational methods). In the same time, recent data sets analysis and machine learning methods have been developed. They are based on graph Laplacian diffusion processes and have been used to perform data sets classification [3], [4] or dimensionality reduction [5] problems.

Inspired by continuous regularization and data-dependent function analysis methods, we propose a general discrete regularization framework on weighted graphs of the arbitrary

This work was partially supported under a research grant of the ANR Foundation (ANR-06-MDCA-008-01/FOGRIMMI), and a doctoral grant of the Conseil Régional de Basse-Normandie and of the Cœur et Cancer association in collaboration with the Department of Anatomical and Cytological Pathology from Cotentin Hospital Center. topologies for any multi-dimensional data [6], [7], [8]. Our regularization framework is expressed directly in a discrete setting and unifies continuous regularizations and graph Laplacian diffusion methods. It provides a formulation for a wide range of applications in various domain. In this paper, we focus on image segmentation and classification problems.

Numerous label diffusion based methods for image segmentation have been proposed, for instance see [9], [10]. If we consider an image as a set of pixels, graph based Laplacian classification is difficult to use due to the great mass of data to analyze. In this work, we propose a fast label diffusion image segmentation scheme. This fast segmentation is performed with a simplified version of the original image. This preprocessing is achieved by a graph based image simplification inspired by the notions of energy partition and the Voronoï diagram [11]. This image simplification, in connection with our graph based regularization, provides a fast, simple and iterative scheme to perform an efficient image segmentation and classification. Moreover, our approach has the advantage to work with graphs of the arbitrary topologies. If we use the fully connected graph on the simplified image version, our framework provides a natural formulation of a fully non-local label diffusion process in contrast with the usual methods.

This paper is organized as follow. In Section II, we recall some definitions and notations on weighted graphs. In Section III, we present the notion of graph energy partitions. It constitutes the basis of our image simplification strategy. In Section IV, we introduce our general discrete regularization framework and its application in cytological image segmentation and classification. On the one hand, semi-supervised classification is considered. On the other hand, a scheme to transform the semi-supervised learning into an unsupervised one is also described. Interesting experiments are shown and notably the ability of our approach to segment non spatially connected elements by using a minimal number of initial labels. Finally, main ideas and future works are summarized in Section V.

\section{Preliminaries on Weighted GraphS}

We consider the general situation where a set of data $X=$ $\left\{x_{1}, \ldots, x_{n}\right\}$, with $x \in \mathbb{R}^{m}$, is described by a finite weighted graph $G=(V, E)$. 
A graph $G=(V, E)$ is composed of a finite set $V$ of vertices describing a set of objects, such as image pixels, image regions, data features, etc. Each vertex $v \in V$ corresponds to an element $x \in X$. $G$ is also consists in a subset $E \subseteq V \times V$ of edges. An edge represents the pairwise relation between two vertices of the graph. In this work, we consider the graph $G$ as weighted, connected, undirected and with no self loops. A undirected graph is weighted when there is a function, called weight function, $w: E \rightarrow \mathbb{R}_{+}$. This function associates a real value $w(u, v)$ to each edge $(u, v) \in E$. It reflects the similarity between vertices $u$ and $v$ and satisfies: $w(u, v)=0$ if $(u, v) \notin E$, and $w(u, v)=w(v, u), \forall(u, v) \in E$.

Let $\mathcal{H}(V)$ be the Hilbert space of functions, where each function $f: V \rightarrow \mathbb{R}^{m}$ assigns a real value $f(v)$ to each vertex $v \in V$. The function $f$ can be thought as a $m$-dimensional feature vector. Similarly, one can define $\mathcal{H}(E)$, the Hilbert function space on edges. These two spaces are endowed with the usual inner product.

The graph representation provides a natural and an useful formulation to model any general discrete data. In image processing, this structure is commonly used to represent digital image. We can quoted some of the commonly used graph structures:

- The uniform sampled grid graph where each vertex represents a image pixel and the edges represent the pixel adjacency relationship (commonly in 4 or 8-connectivity).

- The region adjacency graph where vertices describe the image regions, i.e. the high level structures of the picture, and the graph edges represents the region adjacency relationship.

- The proximity graphs: for instance the $k$-nearest neighbor graph, where each vertex is associated with a set of $k$ close vertices depending on a similarity criterion.

\section{GRAPh BASEd EnERgy PARTITIONS For DATA SIMPLIFICATION}

In cytological images, image pixels are not the single relevant elements. Cells or nuclei can be considered as macro structures of the image, i.e. a group of pixels. A pre-processing step, also called pre-segmentation, can be considered. This approach consists in seeking more local and homogenous components than image pixels but also to respect the geometrical structures of elements contained in the image. Moreover, this data simplification reduces the number of objects to analyze in a post-processing step. To perform this decomposition, one can use mathematical morphology operators such as watersheds or the normalized cuts technics. In this work, we propose to use a graph based approach inspired by energy partitions and Voronoï diagram. The Voronoï diagram is a well-known structure in image processing. It is the result of an image space decomposition into a discrete set of objects. Starting from a set of seeds $S$, each data is associated to the closest seed using a metric function $d$.
Let $G=(V, E)$ be a graph, $V$ be the set of the graph vertices and $S=\left\{s_{i}\right\} \subseteq V$ a set of $K$ seeds over $G$, where $i \in[1, K]$. Let $f: V \rightarrow \mathbb{R}^{m}$ be a function which associates a real-valued vector $f(v)$ to each $v \in V$. Then, a metric $d$ : $V \times V \rightarrow \mathbb{R}_{+}$, for $u \in V$ and $v \in V$, can be defined as:

$$
d(u, v)=\min _{\rho(u, v)} \sum_{\rho(u, v)}\|f(u)-f(v)\|,
$$

where $\rho(u, v)$ represents all the paths over $G$ connecting $u$ to $v$ and $\|$.$\| is the usual Euclidian norm in \mathbb{R}^{m}$.

The energy $d_{S}: V \rightarrow \mathbb{R}_{+}$induced by the metric $d$ for all the seeds of $S$ can be expressed as:

$$
d_{S}(v)=\min _{s_{i} \in S} d_{s_{i}}(v)=\min _{s_{i} \in S} d\left(s_{i}, v\right), \quad \forall v \in V .
$$

The influence zone $z$, also called Voronoï region of a given seed $s_{i}$, is the set of vertices which are closer to $s_{i}$ than to any other seeds. It can be defined, $\forall j \in[1, K]$ and $j \neq i$, as:

$$
z\left(s_{i}\right)=\left\{v \in V: d_{s_{i}}(v) \leq d_{s_{j}}(v)\right\} .
$$

The energy partitions of $G$, for a given set of seeds $S$ and a metric $d$, is the set of influence zones noted by:

$$
Z(S, d)=\left\{z\left(s_{i}\right)\right\}, \quad \forall s_{i} \in S .
$$

For a given graph $G$, to find the energy partitions corresponds to seek a minimal cost path over $G$. Among the graph algorithms dedicated to this shortest path problem, the Dijkstra algorithm can be applied.

Let $f: \Omega \subset \mathbb{Z}^{2} \rightarrow \mathbb{R}^{3}$ be a color image. We define $f$ : $V \rightarrow \mathbb{R}^{3} \in \mathcal{H}(V)$ and $G=(V, E)$, the associated uniform sampled grid graph in 8-connectivity, where each vertex $v$ of $V$ corresponds to a pixel of the image $f$. A pre-segmentation of $f$ corresponds to find the energy partitions of $G$ from a set of seeds $S \subseteq V$, i.e. a graph $G^{\prime}=\left(V^{\prime}, E^{\prime}\right)$ such as:

$$
\left\{\begin{array}{l}
V^{\prime}=S \\
E^{\prime}=\left\{\left(s_{i}, s_{j}\right): z\left(s_{i}\right) \cap z\left(s_{j}\right) \neq \varnothing, i \neq j \quad \forall s_{i}, s_{j} \in S\right\} .
\end{array}\right.
$$

The graph $G^{\prime}$ is an approximation of the image by assigning a model for each influence zone of $Z$. A simple model can be a mean or a median value of each influence zone. This approach is equivalent to a classical watershed algorithm. Computationally, it is faster than watershed by using Dijkstra algorithm and a Fibonacci heap structure rather than a simple priority queue.

Fig. 1 illustrates the application of graph based energy partitions to simplify cytological images. To obtain a set of seeds, the extrema of $f$ can be used. Original images have 134400 pixels. The obtained number of influence zones corresponds approximatively to $5 \pm 2 \%$ of the original ones. The energy images show the ability of this method to respect objects structure by preserving edge information. 

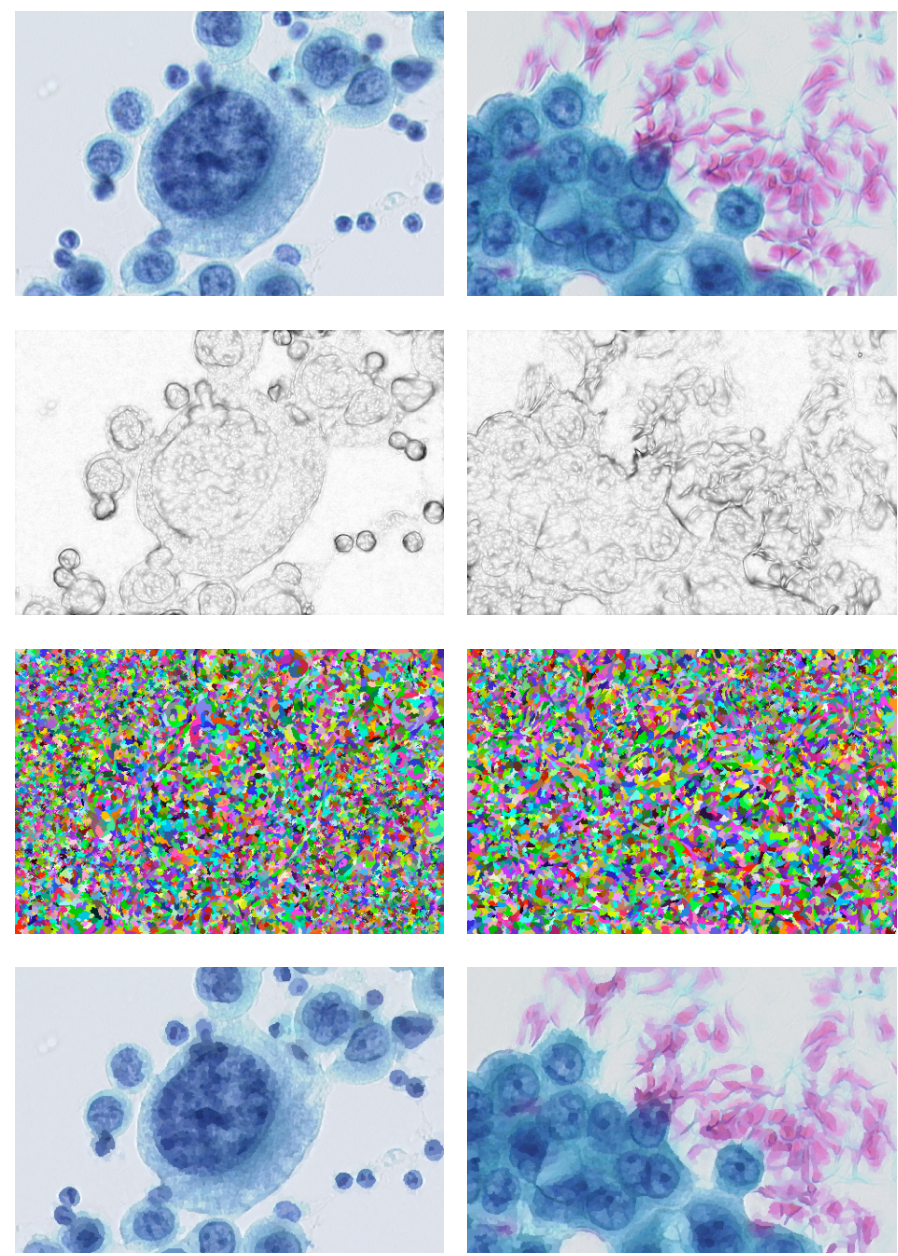

Fig. 1. Cytological image simplification by graph based energy partitions. First row: original images of size $480 \times 320$ i.e. 134400 pixels. Second row: energy images. Third row: influence zones images, 8319 and 6815 regions from left-to-right images, respectively. Fourth row: reconstructed images from influences zones with mean color as region model.

\section{Segmentation and Classification Based on DIFFUSION PROCESSES ON GRAPHS}

In this section, we introduce our discrete regularization framework and its application for image segmentation and classification. First, we focus on semi-supervised learning and finally we propose a scheme to modify the semi-supervised strategy into a unsupervised one.

\section{A. Discrete Regularization Framework}

Let $G=(V, E)$ be a weighted graph. The regularization of a given function $f^{0} \in \mathcal{H}(V)$ corresponds to seek a function $f \in$ $\mathcal{H}(V)$, sufficiently smooth to respect the graph structure of this graph and also close enough to $f^{0}$. This optimization problem can be expressed as the following energy minimization:

$$
\min _{f \in \mathcal{H}(V)}\left\{E_{p}=\sum_{v \in V}\|\nabla f(v)\|^{p}+\lambda \sum_{v \in V}\left\|f-f^{0}\right\|^{2}\right\} .
$$

The first term is the regularizer, also called the smoothness term. The second term is the fitting term. The parameter $p \in$ $[1,+\infty)$. The parameter $\lambda \in[0,+\infty)$ specifies the trade-off between the two competing terms. $\|\nabla f(v)\|$ is the norm of the weighted gradient operator $\nabla f$ at vertex $v$.

Using standard arguments in convex analysis, (1) has a unique solution for $p=1$ and $p=2$ and satisfies, $\forall v \in V$ :

$$
\left.\frac{\partial E_{p}}{\partial f}\right|_{v}=\left(\Delta_{p} f\right)(v)+2 \lambda\left(f(v)-f^{0}(v)\right)=0,
$$

where $\left(\Delta_{p} f\right)(v)$ is the weighted graph p-Laplace operator $\Delta_{p}: \mathcal{H}(V) \rightarrow \mathcal{H}(V)$ of $f$ at the vertex $v$.

In this work, we restrict ourselves to the case of $p=2$. More details on the case of $p=1$ and the defined weighted graph operators can be found in our previous works [8], [12]. In the case of $p=2,\left(\Delta_{p} f\right)(v)$ corresponds to the classical graph Laplacian operator defined as:

$$
(\Delta f)(v)=\left(\Delta_{2} f\right)(v)=2 \sum_{u \sim v} w(u, v)(f(v)-f(u)),
$$

where $u \sim v$ means that vertices $u \in V$ and $v \in V$ are connected by an edge $(u, v) \in E$. Using (3) in (2), the system of equations can be rewritten as:

$$
\left(\lambda+\sum_{u \sim v} w(u, v)\right) f(v)-\sum_{u \sim v} w(u, v) f(u)=\lambda f^{0}(v) .
$$

Among the existing methods, the Gauss-Jacobi iterative algorithm can be applied to resolve (4). Given an iteration step $t$ and an initial function $f^{0}$, the corresponding linearized regularization algorithm is defined, $\forall v \in V$ as:

$$
\left\{\begin{aligned}
f^{0} & =f \\
f^{t+1}(v) & =\frac{1}{\lambda+\sum_{u \sim v} w(u, v)}\left(\lambda f^{0}(v)+\sum_{u \sim v} w(u, v) f^{t}(u)\right) .
\end{aligned}\right.
$$

\section{B. Graph Based Diffusion Processes for Semi-Supervised Classification}

The objective of cytological image analysis is to segment cells from background and/or distinguish cytoplasm from cells' nuclei. Inspired from recent graph based semi-supervised learning [9], [13], we propose to use our discrete regularization framework to perform the semi-supervised classification problem.

A typical semi-supervised learning problem can be described as follow. Given a set of data $V$ composed of labeled and unlabeled data points, the general goal is to estimate the unlabeled points from the labeled ones by a functional regularization based on diffusion processes. In this work, we consider this learning problem and propose to solve it by using the previously formulated regularization framework (5).

Let $V=\left\{v_{1}, \ldots, v_{n}\right\}$ be a set of vertices defined in $\mathbb{R}^{m}, f$ be a function which associates a feature vector to each $v \in V$ and $c_{i}$ be the set of vertices which belong to the $i^{\text {th }}$ class. 
The set $C=\left\{c_{i}\right\}$, with $i \in[1, k]$, is the initial set of all labeled vertices. The initial unlabeled vertices set is the $\{V \backslash$ $C\}$. The learning problem is composed of $k$ classes. Thus, $k$ independent iterative regularization processes are considered, one per class. This is equivalent to define the function $f^{0}$ : $V \rightarrow \mathbb{R}^{k}$, for each regularization process, as:

$$
f_{i}^{0}(v)= \begin{cases}+1 & \text { if } v \in c_{i} \text { with } i \in[1, k], \forall c \in C \\ -1 & \text { otherwise } \\ 0 & \text { if } v \in\{V \backslash C\}\end{cases}
$$

Then, each regularization process follows the same previously defined algorithm (5), with $i \in[1, k]$ and for each $v \in V$ :

$$
f_{i}^{t+1}(v)=\frac{1}{\lambda+\sum_{u \sim v} w(u, v)}\left(\lambda f_{i}^{0}(v)+\sum_{u \sim v} w(u, v) f_{i}^{t}(u)\right),
$$

One can note that the label diffusion algorithm only depends on the estimated labels in the neighborhood of the considered vertex $v$. At the end of the regularization processes, a class is assign to a vertex $v \in V$ by using the following decision function:

$$
c(v)=\operatorname{argmax} \frac{f_{i}(v)}{\sum_{i} f_{i}(v)} .
$$

To construct the representative graph, we propose to use one of the following two similarity functions between two neighbor vertices $u \in V$ and $v \in V$ (but any other formulation can be considered) $: w(u, v)=\exp \left(-h\left(\|u-v\|^{2}\right)\right)$ and $w(u, v)=$ $\frac{1}{\|u-v\|+\epsilon}, \forall(u, v) \in E$, where $h$ is a parameter depending on the variation of $\|u-v\| . \epsilon \rightarrow 0$ is a fixed parameter to avoid the zero denominator. $\|$.$\| is the norm between u$ and $v$ defined on $\mathbb{R}^{m}$.

In image segmentation, it is natural to represent the image as an uniform sampled grid graph (in 4 or 8-connectivity) where each vertex corresponds to an image pixel. But, as mentioned previously, in cytological image, pixels rarely represent relevant elements such as cells or nuclei. Therefore, to construct the weighted graph, we use a pre-segmented image obtained with our graph based image simplification scheme. One way to construct the graph from this simplified image is, for instance, to use the well-known region adjacency graph. Another way is to construct the fully connected graph.

The fully connected graph has the advantage to extend the notion of proximity between two vertices. Two vertices can be similar relatively to the weight function even if they are not spatially close or adjacent in an image. Thus, in connection with the diffusion processes, objects can be quickly labeled as the same class even if they are not spatially adjacent. If we consider an image as a set of pixels, it is clear that this approach can not be applied due to the computing time. But, if we consider the simplified version of images, this method becomes an efficient one. Moreover, this graph structure in connection with the proposed regularization framework, provides a natural fully non-local label propagation process in contrast to the usual methods.

Fig. 2 demonstrates the efficiency of the proposed semisupervised classification algorithm to segment image background and different kinds of biological elements: cytoplasm and nuclei. This example shows a five classes classification problem such as the background, two kinds of cytoplasm and two kinds of nucleus. One can note in Fig. 2(a) that not all relevant elements are marked. A fully connected graph is constructed from Fig. 2(b): the influence zones image. The advantage of the fully connected graph, associated with the proposed label diffusion process, lies in the ability to segment non spatially adjacent regions. The user only needs to label some relevant elements of the image. For instance, in Fig. 2(a), user does not mark the two different cytoplasm but the label diffusion algorithm found all the similar ones relatively to the marked ones. Fig. 2(c) shows the fully non-local label diffusion result after only one iteration.

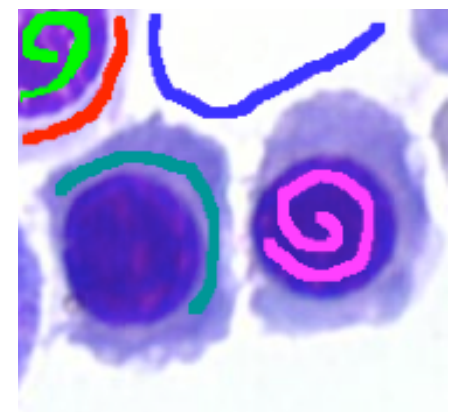

(a)

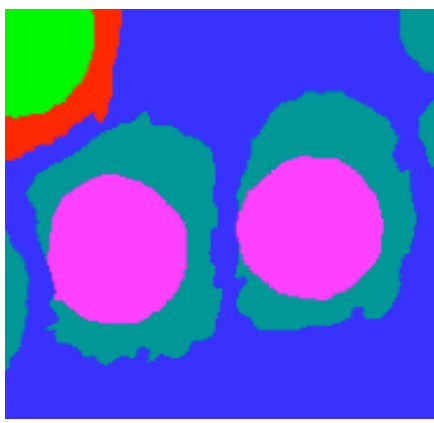

(c)

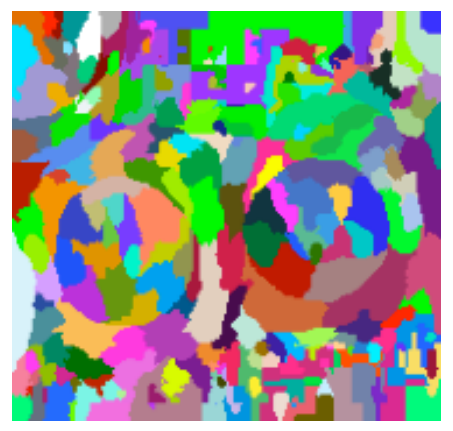

(b)

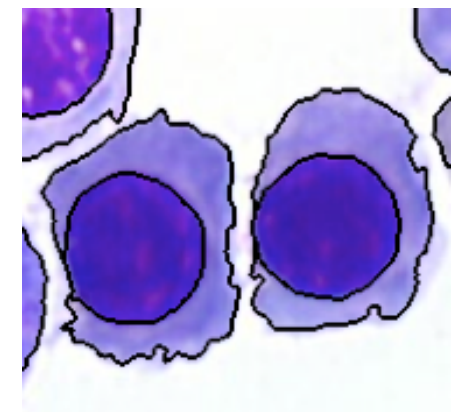

(d)
Fig. 2. 5 classes cytological semi-supervised image segmentation problem. (a) Original image with user initial labels. (b) Influence zones image. (c) Regions map after label diffusion. (d) Original image with the obtained region boundaries superimposed in black color.

This experiment shows the advantage of using the fully connected graph. This structure contains all the image data information. Therefore, only few labels are needed to start the segmentation method. The proposed diffusion algorithm labels objects depending on the closeness defined by the similarity function $w$. A drawback of using the fully connected graph is when the number of vertices increases (a several thousands). It 
will become difficult to obtain results in reasonable computing time (less than a few minutes).

Graphs structures typically used in the literature include fully connected graph, fixed grid graph, or nearest neighbor graph ( $k-$ nn graph, $\epsilon$-graph). Recent studies take an interest in the graph construction itself to optimize the classification computational time. These methods [14] use derivative forms of neighborhood graphs or minimum spanning trees (MST). A MST is a well-known structure in graph theory. It consists in a tree subgraph that contains all the vertices and has a minimum sum of edges weights. This compact graph structure has good properties for our problem. It gives a connected graph with no cycles and is also sparse: for $N$ data points, it has only $N-1$ edges.

Fig. 3 shows the segmentation comparison between fully connected and MST graphs and the computing time for approximatively the same segmentation results. In this example, one can note the influence of the graph structure on the computing time and the segmentation results. Moreover, one can see that user only labels one nucleus as initial label and the diffusion process has found all the nuclei contained in the image.

Choosing the graph structure is an application dependent problem and no general rules can be given. For instance, the MST graph or other proximity graphs speed up the diffusion processes but need a pre-processing for their construction step, usually a distance estimation over all the graph vertices. This step is not needed for the fully connected graph construction. The choice of the appropriate graph structure depends on a fine compromise between application, results accuracies and computing time.

\section{From Semi-Supervised to Unsupervised Classification}

In this section, we show how with the label diffusion algorithm, semi-supervised classification and energy partitions principle, we can obtain an automatic classification scheme.

The cytological image clustering is usually a three classes classification problem: image background, cytoplasm and nuclei. Knowing the number of classes to extract, the idea is to automatically select and classify image representative instances, with an arbitrary machine learning method. Thus, these selected instances can be used as initial labels for the proposed label diffusion scheme.

Our image pre-segmentation step uses a set of seeds to perform image simplification. The idea is to use this set of seeds as initial labels. To label these seeds in three classes, any machine learning algorithm can be used. Once seeds are labeled, the classification based on the label diffusion algorithm (6) can be applied in the same scheme as semisupervised one.

Fig. 4 illustrates an unsupervised classification experiment. Fig. 4(b) shows the classification of influence zones image seeds by $\mathrm{k}$-means algorithm with $k=3$. Each seed is described by the mean color vector of each region. This

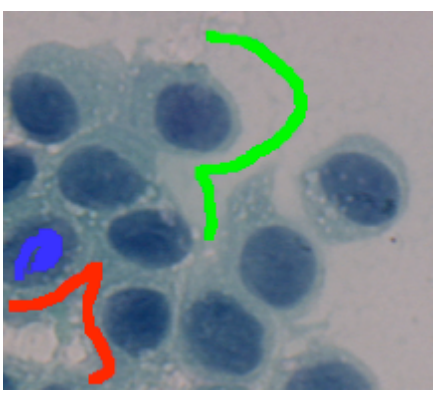

(a)

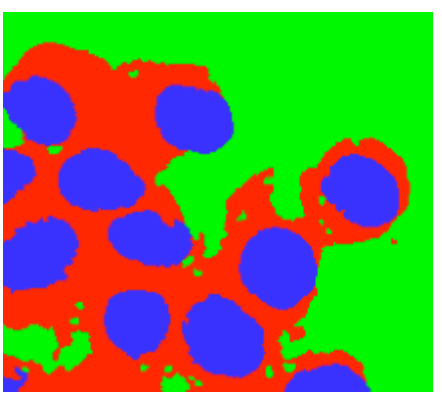

(c)

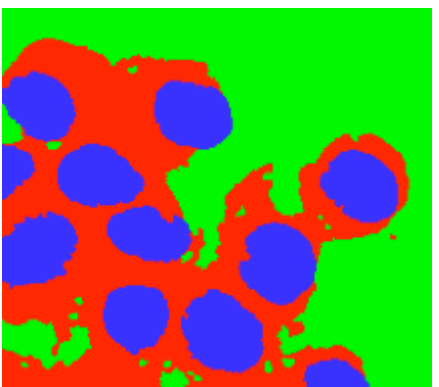

(e)

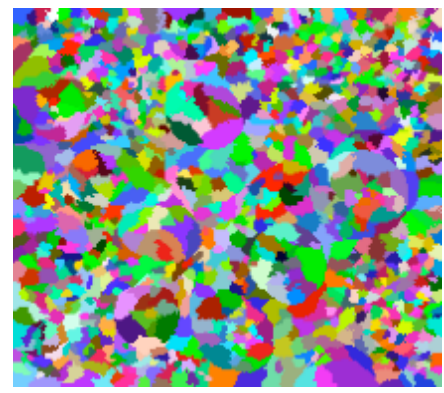

(b)

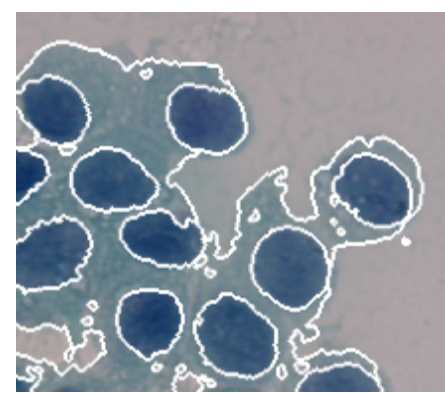

(d)

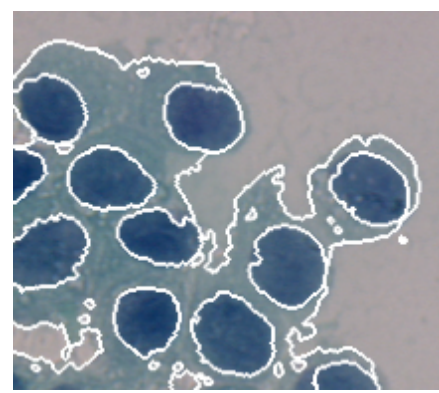

(f)
Fig. 3. Segmentation comparison between fully connected graph and MST graph. (a) User manually labeled image (3 classes problem). (b) Influence zones image. (c) and (e) Regions map after label diffusion. From top-tobottom, results with the fully connected graph obtained in approximatively 5 seconds and results with the MST obtained in less than 1 second. (d) and (f) Original image with the obtained regions boundaries superimposed in white color from (c) and (e), respectively.

example illustrates the ability of the proposed unsupervised classification to extract significant elements. It also shows interesting properties for different values of $\lambda$ parameter in (6). When $\lambda$ is positive value, the label diffusion is highly oriented by initial labels. Obtained results in Fig. 4(c) reflect the initial labels classification. When the $\lambda$ parameter is null, the algorithm has the ability to modify initial labels classification using neighbor ones. Fig. 4(e) shows label modification effects on classification results. Bad initial cytoplasm labels are changed to background label and conversely.

They are differences between a simple region merging algorithm and our automatic classification scheme. Indeed, the proposed method does not need any merging criterion to 
assign a label to a region: it is implicitly done by the weighted graph representation. Moreover, our method does not need any stopping criterion: the final result is obtained when the algorithm reaches the convergence.

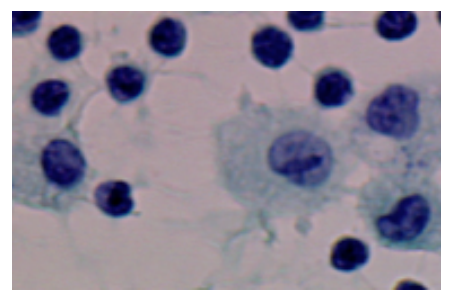

(a)

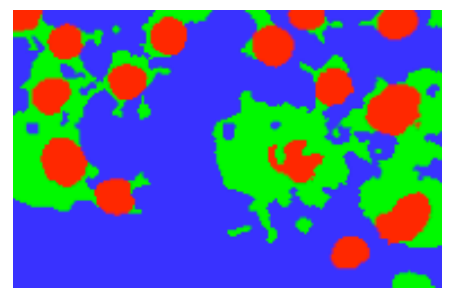

(c)

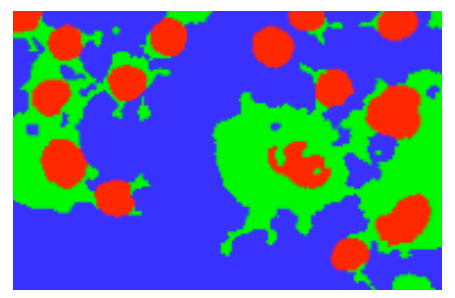

(e)

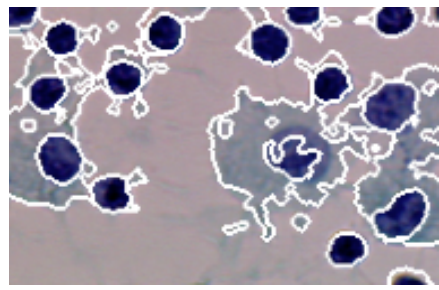

(d)

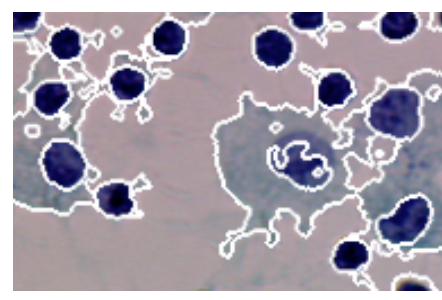

(f)

Fig. 4. Unsupervised classification using energy partitions seeds classified with k-means algorithm. (a) Original image. (b) K-means seeds classification $(k=3)$. (c) and (e) Segmentation regions map with parameter $\lambda=1$ and $\lambda=0$, respectively. (d) and (f) Original image with the obtained region boundaries superimposed in white color from (c) and (e), respectively.

\section{CONCLUSiOn AND Future WORKS}

In this paper, a classification scheme for cytological images is presented. This strategy is based on image simplification and discrete regularization framework. These approaches use graphs as an underlying representation and a unified formulation.

Image simplification has been performed by using graph based energy partitions. This data reduction allows us to efficiently use our discrete regularization based on diffusion processes on graphs to solve cytological image classification problems. The proposed segmentation scheme has the ability to segment elements which are not spatially close or adjacent by using fully connected graphs or MST ones. All the significant elements having to be extracted from an image, do not need to be marked to be segmented by the proposed scheme. Moreover, the fully connected graph, in connection with our regularization framework based on diffusion processes on weighted graphs, provide a natural and uncommon used fully non-local label propagation formulation.

Our work describes two classification approaches. On the one hand, an interesting user guidance interactive semisupervised classification. On the other hand, an unsupervised classification using pre-labeled image seeds. Moreover, our discrete regularization can be also used on any multidimensional data and on any graph of the arbitrary topologies. Thus, our approach can be applied in a wide range of applications in various domains, for instance data sets analysis or clustering. In particular, we plan to categorize and recognize segmented cellular objects in huge image data sets.

\section{REFERENCES}

[1] T. Chan and J. Shen, Image Processing and Analysis: Variational, PDE, Wavelet, and Stochastic Methods. SIAM, 2005.

[2] G. Aubert and P. Kornprobst, Mathematical Problems in Image Processing. Partial Differential Equations and the Calculus of Variations, 2nd ed., ser. Applied Mathematical Sciences. Springer, 2006, vol. 147.

[3] M. Belkin, P. Niyogi, and V. Sindhwani, "Manifold regularization: A geometric framework for learning from labeled and unlabeled examples," Journal of Machine Learning Research, vol. 7, pp. 2399-2434, November 2006.

[4] D. Zhou and B. Schölkopf, Discrete Regularization, Semi-Supervised Learning, ser. Adaptive computation and machine learning. Cambridge, MA, USA: MIT Press, September 2006, section 3.13, pp. 221-232.

[5] S. Lafon and A. B. Lee, "Diffusion maps and coarse-graining: A unified framework for dimensionality reduction, graph partitioning, and data set parameterization," IEEE transactions on Pattern Analysis and Machine Intelligence, vol. 28, no. 9, pp. 1393-1403, September 2006.

[6] V. T. Ta, O. Lezoray, and A. Elmoataz, "Graphs regularization for data sets and images: Filtering and semi-supervised classification," June 2007, PASCAL Workshop: Learning from and with graphs, 6th IAPR-TC-15 Workshop on Graph based Representations in Pattern Recognition.

[7] O. Lezoray, S. Bougleux, and A. Elmoataz, "Parameterless discrete regularization on graphs for color image filtering," in Image Analysis and Recognition, ser. Lecture Notes in Computer Science, M. Kamel and A. Campilho, Eds., vol. 4633. Springer, August 2007, p. to appear.

[8] S. Bougleux, A. Elmoataz, and M. Melkemi, "Discrete regularization on weighted graphs for image and mesh filtering," in Scale Space and Variational Methods in Computer Vision, ser. Lecture Notes in Computer Science, F. Sgallari, A. Murli, and N. Paragios, Eds., vol. 4485. Springer, May 2007, pp. 128-139.

[9] F. Wang, C. Zhang, H. C. Shen, and J. Wang, "Semi-supervised classification using linear neighborhood propagation," in IEEE Computer Society Conference on Computer Vision and Pattern Recognition, ser. IEEE Conference Proceedings, vol. 1. IEEE Computer Society, June 2006, pp. 160-167.

[10] L. Grady, "Random walks for image segmentation," IEEE Transactions on Pattern Analysis and Machine Intelligence, vol. 28, no. 11, pp. 17681778, November 2006.

[11] P. A. Arbeláez and L. D. Cohen, "Energy partitions and image segmentation," Journal of Mathematical Imaging and Vision, vol. 20, no. 1-2, pp. 43-57, January-March 2004.

[12] O. Lezoray, A. Elmoataz, and S. Bougleux, "Graph regularization for color image processing," Computer Vision and Image Understanding, vol. 107, no. 1-2, pp. 38-55, July-August 2007.

[13] A. D. Szlam, M. Maggioni, and R. R. Coifman, "A general framework for adaptive regularization based on diffusion processes on graphs," Yale University, Tech. Rep. 1365, 2006, submitted.

[14] M. Carreira-Perpiñán and R. Zemel, "Proximity graphs for clustering and manifold learning," in Advances in Neural Information Processing Systems 17, Saul, Weiss, and Bottou, Eds. Cambridge, MA, USA: MIT Press, 2005, pp. 225-232. 